\section{Effect of Potassium on Muscle Tension, especially on that of Vascular Muscle}

Is the previous communication it was shown that in corticosteroid-induced hypertension in the rat, of all the electrolyte shifts, only potassium increase in the aorta wall was characteristic. In striated muscle or in other organs containing smooth muscle, the potassium content either decreases or remains unchanged.

It has been assumed that the increase of concentration of potassium in the vascular muscle causes a tension increase and, as a result, high pressure. Because of this the following experiments were done in vitro: (a) the effect of potassium on muscle tension; (b) qualitative differences concerning the effect of potassium on different muscle types were investigated.

The experiments were especially carried out on carotis strips of cattle, on the museulus rect. abd. of the frog and the rat as well as on the muscle of large intestine of the rat in Tyrode bath, at adequate temperature and with known, variable initial tension. The changes in tension were registered with a transducer fitted perpendicularly to the surface of the examined tissue.

If the potassium content of the Tyrode solution is increased, the tension of the vessel strip increases too. Contrary to Raab's findings ${ }^{1}$ the increase of tension can last up to $90 \mathrm{~min}$. according to potassium content and will last as long as potassium is not changed (Fig. 1).

The latent period, the steepness of the tension increase and the total tension depend on the potas-
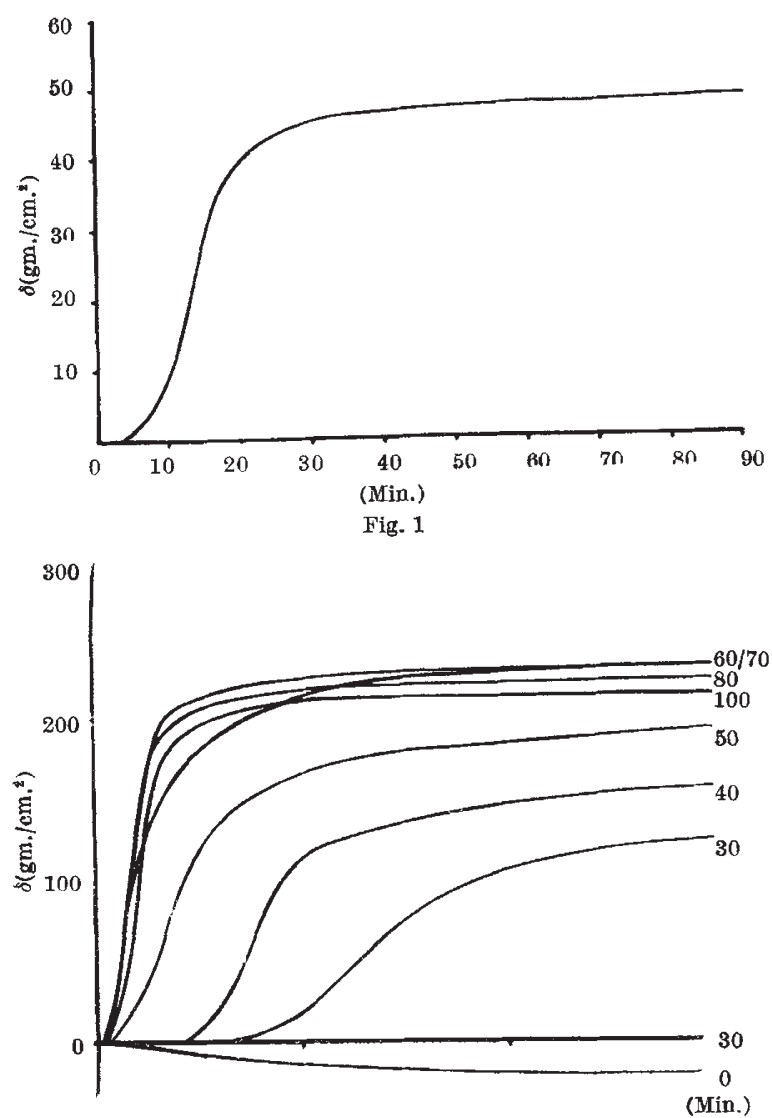

Fig. 2. Course of tension with different concentrations of potassium. Figures on the right indicate $m M[K]$

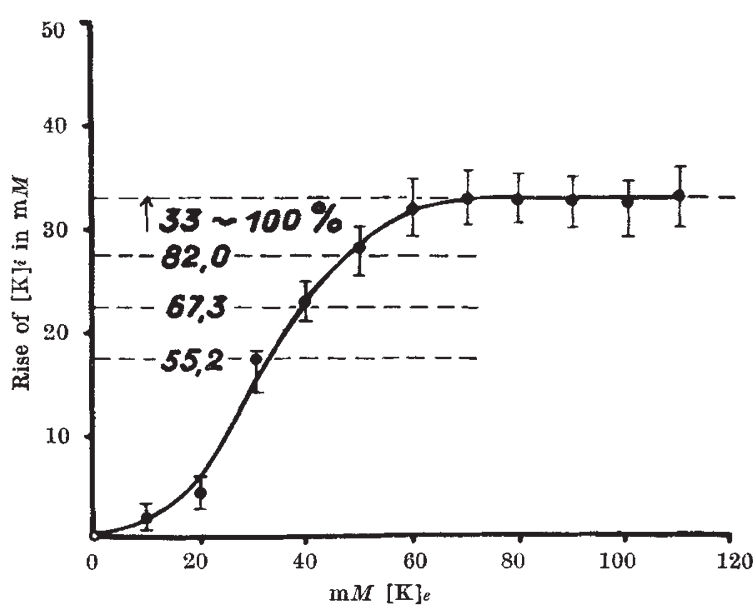

Fig. 3. Abscissa, $\mathrm{m} M$ in Tyrode solution. Ordinate, $\mathrm{m} M[\mathrm{~K}] \mathrm{i}$ increase, after $30 \mathrm{~min}$.

sium of the bath solution. The maximal tension is attained with a concentration of $60-70 \mathrm{~m} M$ (Fig. 2). An equilibrium between the bath solution and the extracellular space is established after about $5 \mathrm{~min}$. The increase in tension initiated by potassium and the decrease after removal of it are not influenced by the oxygen content of the bath solution, as is the case with tension caused by electric stimulation or epinephrine. The influence of potassium on striated muscle or on smooth muscle is different. With skeletal muscle as well as with smooth muscle of large intestine only a temporary increase in tension could be produced with the concentrations used. In the muscle of large intestine $30 \mathrm{~m} M$ potassium first produces an increase in tonus, then a rhythmic fluctuation in tonus.

Since on one hand with high-pressure animals the intracellular potassium in the aortic wall is increased, and on the other, as shown, potassium has different effects according to muscle type, the vascular muscle and the muscle of large intestine were examined in regard to their permeability to potassium. The $[\mathbf{K}]_{i}$ was calculated from the total content of $\mathbf{K}-[\mathbf{K}]$ e. The value of the extracellular space was determined with thiosulphate. As shown in Fig. 3, potassium already penetrates into the vascular muscle when the [K] of the bath solution amounts to $10 \mathrm{mM}$ and saturation occurs with a [K] of $60-70 \mathrm{mM}$. If we set the maximum value of the tension increase (Fig. 2) and the $[\mathrm{K}]_{i}$ (Fig. 3) equal to 100 , it will be found that the relative tension is proportional to the relative $[\mathrm{K}]_{i}$ (Fig. 3 ). The course of penetration of potassium into the muscle fibre and of that of tension are similar within a given concentration of the bath solution. No potassium enters the musele of large intestine of the rat under the same experimental conditions.

It is assumed that two different functional systems are present in the vascular muscle, the contractile system for the transient regulatory tension and the tonic system for the continuous tension. The tension of the contractile system seems to depend on the concentration of potassium in the muscle.

L. LASZT

Department of Physiology,

University of Fribourg,

Fribourg, Switzerland.

${ }^{1}$ Raab, E., Pfiügers Arch., 215, 651 (1927). 